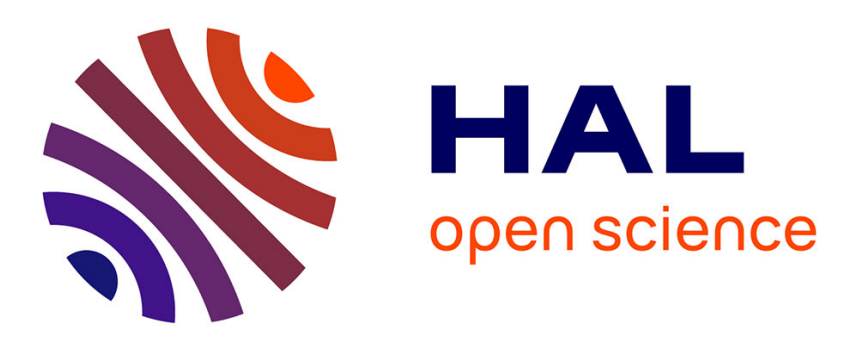

\title{
Influence de la nature et de l'énergie d'un faisceau ionique sur le gain d'un multiplicateur d'électrons utilisé en spectrométrie de masse \\ F. Hoffert, J. Paulus, J.P. Adloff
}

\section{- To cite this version:}

F. Hoffert, J. Paulus, J.P. Adloff. Influence de la nature et de l'énergie d'un faisceau ionique sur le gain d'un multiplicateur d'électrons utilisé en spectrométrie de masse. Revue de Physique Appliquée, 1966, 1 (1), pp.43-47. 10.1051/rphysap:019660010104300 . jpa-00242683

HAL Id: jpa-00242683

https://hal.science/jpa-00242683

Submitted on 1 Jan 1966

HAL is a multi-disciplinary open access archive for the deposit and dissemination of scientific research documents, whether they are published or not. The documents may come from teaching and research institutions in France or abroad, or from public or private research centers.
L'archive ouverte pluridisciplinaire HAL, est destinée au dépôt et à la diffusion de documents scientifiques de niveau recherche, publiés ou non, émanant des établissements d'enseignement et de recherche français ou étrangers, des laboratoires publics ou privés. 


\title{
INFLUENCE DE LA NATURE ET DE L'ÉNERGIE D'UN FAISCEAU IONIQUE SUR LE GAIN D'UN MULTIPLICATEUR D'ÉLECTRONS UTILISÉ EN SPECTROMÉTRIE DE MASSE
}

\author{
Par F. HOFFERT, J. PAULUS et J. P. ADLOFF, \\ Centre de Recherches Nucléaires, Département de Chimie Nucléaire, Strasbourg-Cronenbourg.
}

\begin{abstract}
Résumé. - On étudie les variations de la réponse d'un multiplicateur d'électrons, adapté sur un spectromètre de masse Atlas, en fonction del'énergie (entre 1,2 et $5 \mathrm{keV}$ ), de la masse, du numéro atomique, de la structure et de la charge des ions incidents.
\end{abstract}

Abstract. - The variation of the gain of an electron multiplier, used as a collector on an Atlas mass spectrometer, has been measured as a function of the energy (between 1.2 and $5 \mathrm{keV}$ ), mass atomic number, structure and charge of the incident ions.

Les multiplicateurs d'électrons sont employés en spectrométrie de masse lorsque la sensibilité des collecteurs d'ions ordinaires (10-13 A) s'avère insuffisante, et en particulier pour l'étude de réactions entre ions et molécules ou de processus d'ionisation de faible efficacité. Dans ce type de détecteurs, dont la limite de sensibilité peut atteindre $10^{-10} \mathrm{~A}$, le courant ionique est transformé en courant électronique par l'émission secondaire qui résulte de l'impact des ions sur une surface métallique. Ce courant est amplifié par des dynodes dont l'assemblage est identique aux électrodes des photomultiplicateurs. La réponse du multiplicateur est déterminée par les modalités de la conversion du faisceau ionique en électrons au niveau du premier étage du détecteur.

De manière générale, les mécanismes de l'émission potentielle et cinétique contribuent au coefficient d'émission secondaire $\gamma$ :

$$
\gamma_{\text {total }}=\gamma_{\text {pot. }}+\gamma_{\text {cin }}
$$

Selon Hagstrum [1], le premier terme n'intervient que lorsque la condition $E_{\mathrm{i}}>2 \varphi$ est satisfaite, $E_{\mathrm{i}}$ étant le potentiel d'ionisation de l'ion et $\varphi$ le travail d'extraction de la cible. Parilis et Kishineskii[2] ont établi une expression théorique du coefficient d'émission secondaire $\gamma$ consécutif à l'émission cinétique: il n'apparaît qu'au-dessus d'une certaine vitesse ou énergie de l'ion incident. Il est évident que tous les paramètres susceptibles d'influencer l'émission secondaire peuvent altérer la réponse du multiplicateur.

En 1939, Allen [3], [4] a réalisé un multiplicateur d'électrons à 12 étages et à focalisation électrostatique; les dynodes étaient constituées par un alliage $\mathrm{Cu}$-Be. Les détecteurs de ce type sont actuellement les plus utilisés en spectrométrie de masse. Pour un multiplicateur donné et dans des conditions expérimentales déterminées, la nature de la cible, l'angle d'incidence du faisceau, la tension appliquée entre les différents étages du détecteur et la pression dans l'enceinte du détecteur peuvent être maintenus à une valeur constante. Il est alors possible de déterminer la réponse du multiplicateur en fonction des paramètres du faisceau ionique, c'est-à-dire l'énergie ou la vitesse des particules, ainsi que la nature et la structure électronique des ions incidents.

Dans ce mémoire, nous examinons la réponse du multiplicateur pour des ions d'énergie comprise entre 1,2 et $5 \mathrm{keV}$.

1. Partie expérimentale. - Le spectromètre de masse est un appareil Atlas, type $\mathrm{CH}$ IV. Les multiplicateurs utilisés au cours de ce travail ( ${ }^{\mathbf{1}}$ ) (étudiés par Schaetti [5]) comprennent 17 étages; les dynodes de $\mathrm{Cu}$-Be sont traitées par activation et la focalisation est électrostatique. Le gain du multiplicateur est déterminé à l'aide des valeurs du courant ionique primaire $I_{\mathrm{p}}$ et du courant électronique secondaire $I_{\mathrm{s}}$. Pour que la mesure soit correcte il est nécessaire que la totalité du faisceau ionique soit reçue successivement par la cage de Faraday et la première dynode du multiplicateur d'électrons.

Cette condition est satisfaite dans le montage dont le schéma est représenté ( $f g .1)$. Le système de collection comprend deux fentes d'entrée séparées ; la faible distance entre le collecteur d'ions $C$ et la première dynode du multiplicateur élimine les effets de discrimination " géométrique " importants qui ont été mis en évidence avec le montage original du constructeur.

a) Mesure de $I_{\mathrm{p} .}$ - Elle est réalisée à l'aide de la cage de Faraday $C$ par l'intermédiaire d'une résistance $R_{2}$ de $10^{11}$ ohms ; la fente $S_{2}$ et la première

(1) Ces multiplicateurs ont été réalisés par l'Institut für Technische Physik, E. T. H., Zurich. 


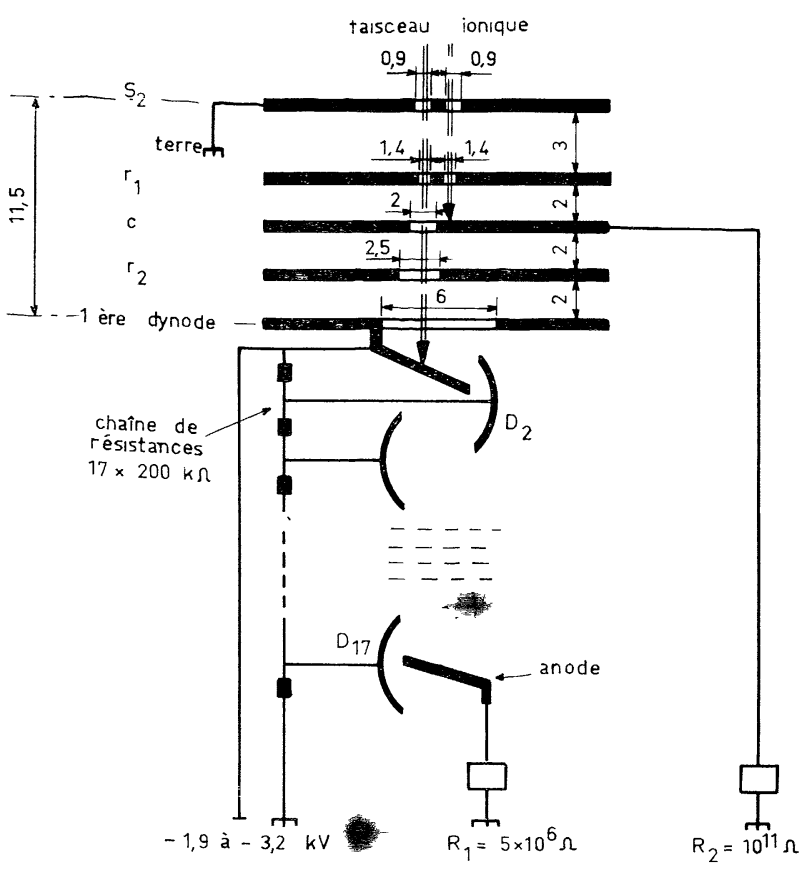

Fig. 1. - Assemblage du collecteur d'ions et du multiplicateur d'électrons (les cotes sont indiquées en $\mathrm{mm}$ ).

dynode du multiplicateur sont au potentiel zéro; le potentiel des repousseurs d'électrons secondaires $r_{1}$ et $r_{2}$ est -85 volts.

b) Mesure de $I_{\mathrm{s}}$. - Le courant électronique secondaire est mesuré à l'anode du multiplicateur d'électrons par l'intermédiaire d'une résistance $R_{1}$ de $5 \times 10^{2}$ ohms. L'anode du détecteur et la fente $S_{2}$ sont au potentie] zéro; les potentiels des repousseurs d'électrons $r_{1}$ et $r_{2}$, du collecteur d'ions $C$ et de la première dynode sont $-1,9 \mathrm{kV}$.

Le gain $G$ du multiplicateur est égal au rapport des courants électronique secondaire $I_{\mathrm{s}}$ et ionique primaire $I_{\mathrm{p}}$ :

$$
G=\frac{I_{\mathrm{s}}}{I_{\mathrm{p}}}=\frac{V_{1}}{V_{2}} \times \frac{R_{2}}{R_{1}}
$$

$V_{1}$ et $V_{2}$ étant les chutes de tension aux bornes des résistances $R_{1}$ et $R_{2}$.

La tension d'accélération effective des particules incidentes est égale à la différence de potentiel entre la source d'ions et la première dynode du multiplicateur. La tension appliquée à la source d'ions est variable entre 1,2 et $3,1 \mathrm{kV}$. Ce dispositif permet d'étudier la réponse du multiplicateur d'électrons pour des ions d'énergie comprise entre 3,1 et $5 \mathrm{keV}$; en portant l'anode du multiplicateur à la tension de $+1,9 \mathrm{kV}$ et la première dynode au potentiel zéro, la gamme des énergies s'étend de 1,2 à $3,1 \mathrm{keV}$. L'emploi successif des deux montages permet d'explorer le domaine d'énergie compris entre 1,2 et $5 \mathrm{keV}$, avec une possibilité de recoupement à $3,1 \mathrm{keV}$ cette valeur pouvant être obtenue par l'un ou l'autre dispositif. L'incertitude sur le gain du multipli. cateur est $\pm 3 \%$.

2. Étude de la réponse du multiplicateur d'électrons. - Le gain du multiplicateur a été mesuré pour un grand nombre d'ions d'énergie égale à 5,4 , $3,1,2,1$ et 1,2 keV. Le recoupement des résultats a permis de dégager l'influence des paramètres caractéristiques des particules incidentes.

A) Énergie et vitesse des ions ( $f$ g. 2.). - Le gain augmente linéairement avec l'énergie des ions ${ }^{4} \mathrm{He}^{+},{ }^{20} \mathrm{Ne}^{+},{ }^{40} \mathrm{Ar}^{+},{ }^{84} \mathrm{Kr}^{+}$et ${ }^{129} \mathrm{Xe}^{+}$. (La même loi est observée pour les ions halogènes ${ }^{19} \mathrm{~F}^{+},{ }^{35} \mathrm{Cl}^{+}$, ${ }^{81} \mathrm{Br}^{+}$et ${ }^{127} \mathrm{I}^{+}$.)

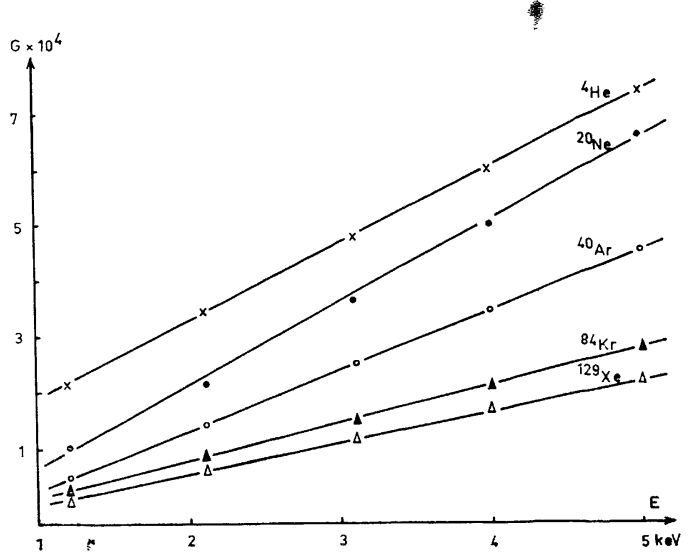

Fig. 2. - Variation du gain avec l'énergie des ions ${ }^{4} \mathrm{He}^{+},{ }^{20} \mathrm{Ne}^{+},{ }^{40} \mathrm{Ar},{ }^{84} \mathrm{Kr}^{+}$et ${ }^{129} \mathrm{Xe}^{+}$.

La variation linéaire du gain avec l'énergie des ions est générale, quelles que soient la nature et la structure des ions et ceci dans le domaine d'énergie compris entre 1,2 et $5 \mathrm{keV}$. Cette conclusion est en bon accord avec les résultats de Michijima [6] relatifs aux ions ${ }^{40} \mathrm{Ar}^{+},{ }^{20} \mathrm{Ne}^{+}$et ${ }^{28} \mathrm{~N}^{+}$

$$
(800<E<1800 \mathrm{eV}) \text {. }
$$

Inghram, Hayden et Hess [7] ont relevé un comportement analogue pour les ions des éléments alcalins $(E<10 \mathrm{keV})$. Cette variation régulière implique une variation similaire du coefficient d'émission secondaire $\gamma$ dans le domaine des faibles énergies, observée effectivement par Higatsberger, Demorest et Nier [8] pour plusieurs ions ( $1<E<6 \mathrm{keV})$.

Il est possible de confronter ces résultats avec la théorie de Parilis et Kishineskii [2]. La variation du coefficient $\gamma$ est déterminée essentiellement par celle de la section efficace effective d'ionisation $\sigma^{*}(v)$, où $v$ est la vitesse de l'ion incident. Pour les vitesses ioniques comprises entre $10^{7}$ et $3 \times 10^{7} \mathrm{~cm} / \mathrm{s}$ on a

$$
\sigma^{*}(v) \approx v^{2}-\left(\frac{3}{2} v_{\min .}\right)^{2}
$$


$v_{\text {min. }}$ représente le seuil de vitesse de l'émission cinétique. C'est le domaine de variation linéaire de $\gamma$ avec l'énergie de l'ion incident. La relation précédente est valable lorsque $\left[(1 / 4)<\left(Z_{1} / Z_{2}\right)<4\right]$ où $Z_{1}$ et $Z_{2}$ sont respectivement les numéros atomiques de la cible et de l'ion incident. Elle ne s'applique donc, dans notre cas, qu'aux ions ${ }^{12} \mathrm{C}^{+}$ à ${ }^{129} \mathrm{Xe}^{+}$dont les vitesses sont comprises entre les valeurs limites $v_{1}=2,83 \times 10^{7} \mathrm{~cm} / \mathrm{s}$ (vitesse de ${ }^{12} \mathrm{C}^{+}$à $5 \mathrm{keV}$ ) et $v_{2}=0,42 \times 10^{7} \mathrm{~cm} / \mathrm{s}$ (vitesse de ${ }^{129} \mathrm{Xe}^{+}$à $\left.1,2 \mathrm{keV}\right)$. La théorie de Parilis et Kishineskii prévoit précisément une variation linéaire de $\gamma$ avec l'énergie des ions, en accord avec les valeurs mesurées de $G$.

Les pentes des droites de la figure 2 diminuent avec l'augmentation de la masse de l'ion, mais aussi de son numéro atomique.

B) Nature de L'ion. - Le rôle principal dans le processus de l'émission électronique secondaire a été longtemps attribué à la masse de la particule incidente; de récents travaux ont montré l'influence de la couche électronique de l'ion incident. Nous examinerons en premier lieu les ions atomiques afin de déterminer successivement l'influence de la masse $m$, puis du numéro atomique $Z$ de l'ion.

1. Ions atomiques : a) Influence de la masse. Celle-ci est mise le plus sûrement en évidence en considérant les valeurs de $G$ pour les différents isotopes d'un même élément : on voit sur la figure 3 ,

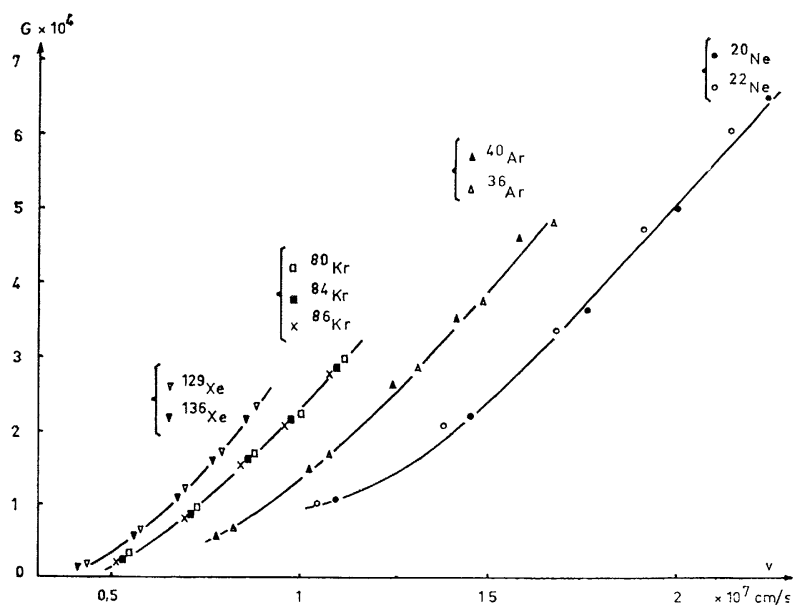

Fig. 3. - Variation du gain avec la vitesse d'ions isotopiques.

représentant la variation du gain avec la vitesse des ions incidents, que tous les isotopes se situent sur la même courbe. Une observation similaire a été faite pour les isotopes du mercure, du chlore et du brome.

Ploch et Walcher [9], en étudiant les couples isotopique suivants : ${ }^{6} \mathrm{Li}^{+} \longrightarrow 7 \mathrm{Li}^{+},{ }^{20} \mathrm{Ne}^{+}-{ }^{22} \mathrm{Ne}^{+}$, ${ }^{39} \mathrm{~K}^{+}-{ }^{41} \mathrm{~K}^{+}$et Higatsberger, Demorest et Nier [8] pour ${ }^{20} \mathrm{Ne}^{+}-{ }^{22} \mathrm{Ne}^{+}$et ${ }^{36} \mathrm{Ar}^{+}-{ }^{40} \mathrm{Ar}^{+}$trouvent, à vitesse égale, un coefficient d'émission secondaire $\gamma$ identique pour les différents isotopes d'un même élément.

Cependant les calculs théoriques de Parilis et Kishineskii prévoient un affet de la masse, même à vitesse égale

$$
-\frac{\Delta \gamma}{\gamma} \frac{m}{\Delta m}=\frac{M}{\sigma^{*}} \frac{\mathrm{d}}{\mathrm{d} k}\left(\Delta \sigma \frac{\mathrm{d} k}{\mathrm{~d} m}\right)
$$

avec

$$
k=\frac{2,48 \pi N a_{0} e^{2} Z_{1} Z_{2}}{\left(\sqrt{Z_{1}}+\sqrt{Z_{2}}\right)^{2 / 3}(m+M)} \quad a_{0}=\hbar^{2} / m e^{2}
$$

$m$ et $M$ représentent les masses de l'ion incident de charge nucléaire $Z_{1}$ et de la cible de numéro atomique $Z_{2} ; N$ est le nombre d'atomes par $\mathrm{cm}^{3}$ de la cible. L'effet de masse a été mis en évidence par Brunnee [10] pour les isotopes des ions alcalins. Nos résultats peuvent difficilement être confrontés avec les prévisions théoriques en raison de l'incertitude sur la valeur de la masse de l'élément-cible. Les relations sont vérifiées au moins qualitativement puisqu'on observe une différence de gain pour les ions isotopiques de même énergie; le gain est toujours plus faible pour l'ion le plus lourd. A vitesse égale, l'effet isotopique, théoriquement attendu, est faible et se trouve estompé par les erreurs expérimentales.

En résumé, pour des ions de même vitesse, le gain du multiplicateur est, en première approximation indépendant de la masse. Sugiura [11] est arrivé à une conclusion identique.

b) Influence de la structure électronique. - I a théorie de Parilis et Kishineskii met l'accent sur le rôle important du numéro atomique $Z$ de l'ion incident. L'expression de la section efficace d'ionisation comprend le terme :

$$
f\left(Z_{1}, Z_{2}\right)=\left(\frac{Z_{1}+Z_{2}}{\overline{\sqrt{Z_{1}}+\sqrt{Z_{2}}}}\right)^{2} .
$$

L'influence de $Z$ devient de plus en plus nette lorsque la vitesse de la particule augmente.

Il a été possible de représenter la variation du gain $G$ en fonction de $Z$ pour 3 vitesses des ions incidents ( $f$ g. 4 ) ; bien que la gamme des vitesses ne soit pas étendue on voit que l'effet de $Z$ devient de plus en plus important lorsque la vitesse $v$ de l'ion croît. Cette conclusion est en accord avec les considérations théoriques déjà citées.

2. Ions moléculaires. - Les gains mesurés pour des ions polyatomiques sont supérieurs aux valeurs obtenues pour des ions monoatomiques de masses voisines et de même énergie.

Ce résultat a déjà été signalé par Stanton, Chupka et Inghram [12] qui ont trouvé :

$$
\gamma\left(\mathrm{CH}_{2}^{+}\right)>\gamma\left(\mathrm{N}^{+}\right) \text {et } \gamma\left(\mathrm{C}_{14} \mathrm{H}_{10}^{+}\right) \simeq 4 \gamma\left({ }^{178} \mathrm{Hf}+\right) \text {. }
$$




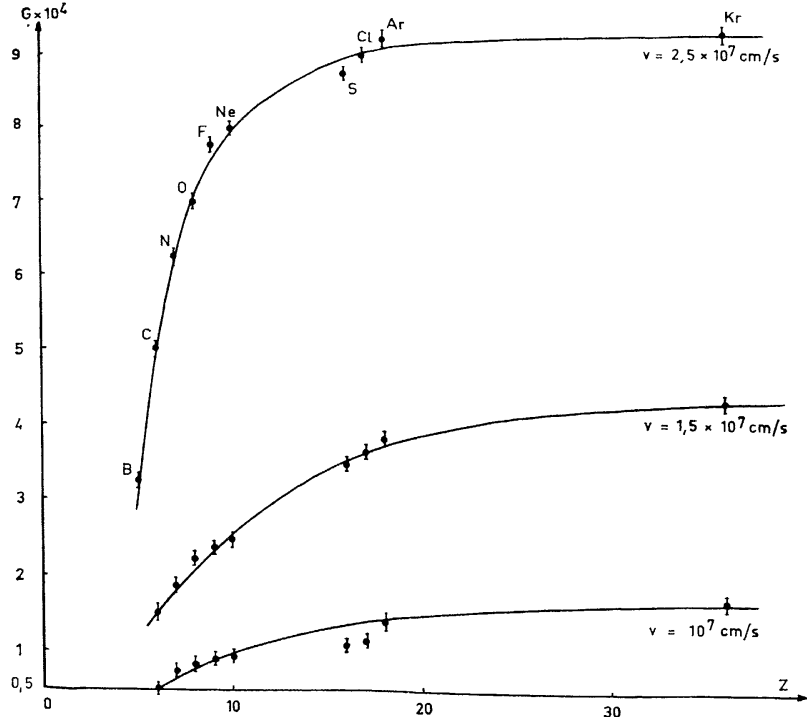

FIG. 4. - Variation du gain avec le numéro atomique.

La figure 5 représente la variation du gain en fonction de la vitesse des ions du spectre de masse du méthane $\left(10^{7}<v<3 \times 10^{7} \mathrm{~cm} / \mathrm{s}\right)$. Les courbes relatives aux ions $\mathrm{C}^{+}, \mathrm{CH}^{+}, \mathrm{CH}_{2}^{+}, \mathrm{CH}_{3}^{+}, \mathrm{CH}_{4}^{+}$se rejoignent pour une vitesse de l'ordre de

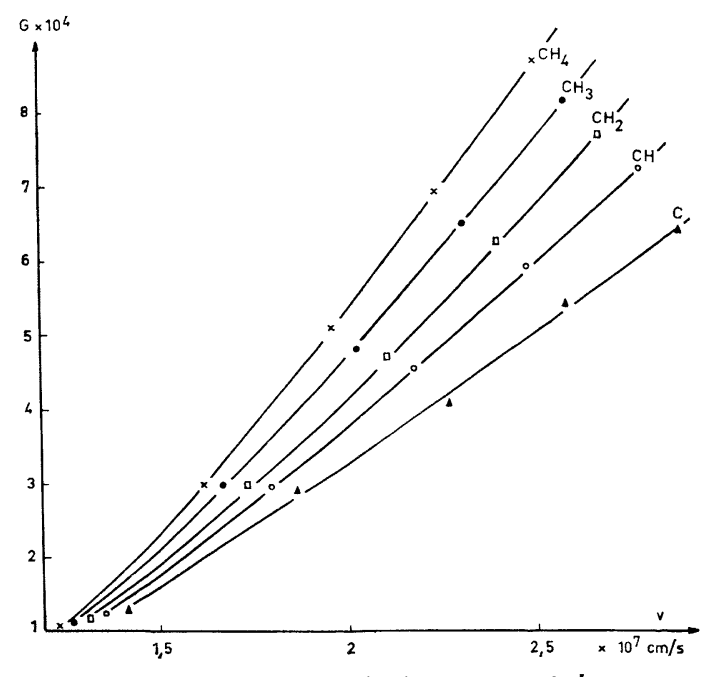

FIg. 5. - Variation du gain:

avec la vitesse des ions $\mathrm{C}^{+}, \mathrm{CH}^{+}, \mathrm{CH}_{2}^{+}, \mathrm{CH}_{3}^{+}$et $\mathrm{CH}_{4}^{+}$.

$1,2 \times 10^{7} \mathrm{~cm} / \mathrm{s}$. La structure moléculaire n'a alors aucune influence sur.le gain du multiplicateur. Pour des vitesses plus élevées, les courbes divergent nettement et pour une même vitesse on a tounours :

$G\left(\mathrm{CH}_{4}^{+}\right)>G\left(\mathrm{CH}_{3}^{+}\right)>G\left(\mathrm{CH}_{2}^{+}\right)>G\left(\mathrm{CH}^{+}\right)>G\left(\mathrm{C}^{+}\right)$

Il est plausible qu'un ion moléculaire d'énergie suffisante soit dissocié lors de la collision avec la cible en un certain nombre de fragments d'énergie inférieure à celle de l'ion incident.
Cas particulier : Série des hydrocarbures.

La figure 6 représente l'évolution du gain en fonction de la vitesse pour des ions de structures moléculaires différentes. Les ions ayant le même nombre d'atomes se placent sur la même courbe ; dans le domaine de vitesse compris entre $1,2 \times 10^{7}$ et $2,8 \times 10^{7} \mathrm{~cm} / \mathrm{s}$ (les ions étant accélérés à $5 \mathrm{keV}$ ) le gain relatif à un ion moléculaire est d'autant plus élevé qu'il contient plus d'atomes, à vitesse égale.

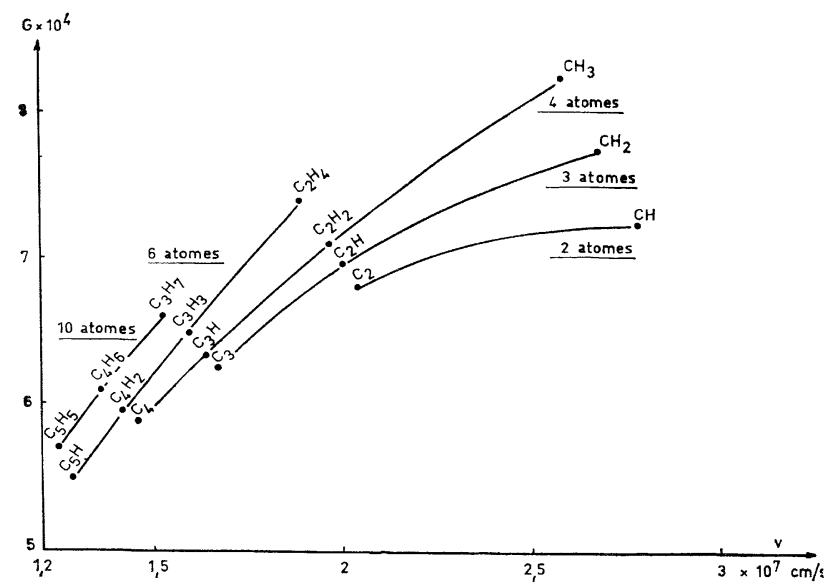

Frg. 6. - Variation du gain avec la vitesse des ions polyatomiques d'énergie $5 \mathrm{keV}$.

Par contre, pour une énergie de 1,2 keV, tous les ions formés par dissociation se rangent sur la même droite, quelle que soit leur structure.

C) Influence de la charge. - Le rôle de la charge ionique sur le gain du multiplicateur est

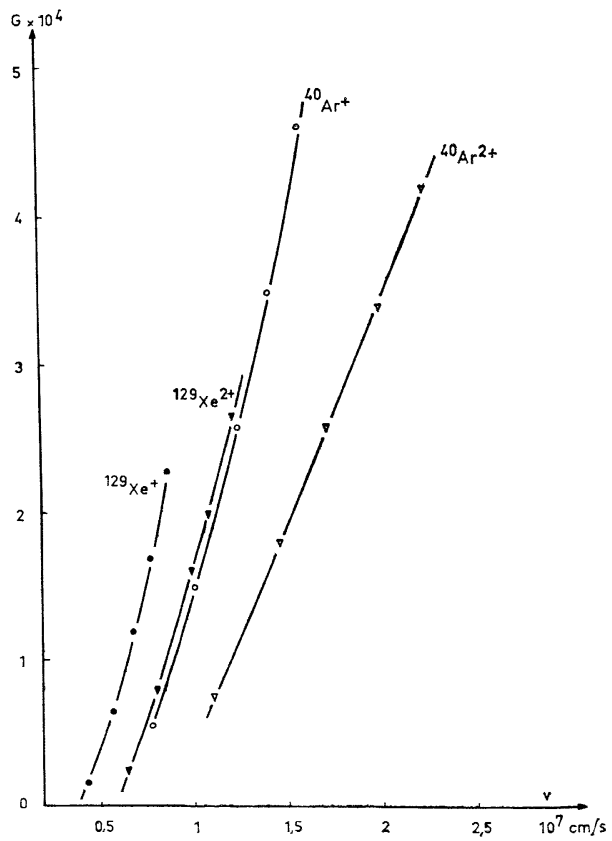

Frc. 7. - Variation du gain avec la charge de l'ion. 
encore mal connu, les résultats trouvés dans la littérature sont souvent divergents, voire même contradictoires.

La théorie de Parilis et Kishineskii ne fait pas intervenir la charge. Ceci a été confirmé expérimentalement pour $\mathrm{Ar}^{+}, \mathrm{Ar}^{2+}$ et $\mathrm{Ar}^{3+}$ : pour une même vitesse des ions, les gains mesurés sont identiques [13]. La figure 7 représente l'évolution des gains pour des ions à charge simple et double en fonction de la vitesse des particules.

$A$ vitesse égale, quelles que soient la nature et la structure de l'ion incident, le gain mesuré pour les ions de charge $+2 \mathrm{e}$ est toujours inférieur au gain déterminé pour des ions de charge + e. Ces résultats sont en accord avec ceux de Higatsberger, Demorest et Nier [8] qui ont bombardé une cible de $\mathrm{Cu}-\mathrm{Be}$ par les ions ${ }^{40} \mathrm{Ar}^{+}-{ }^{40} \mathrm{Ar}^{2+}$ et ${ }^{202} \mathrm{Hg}^{+}-{ }^{202} \mathrm{Hg}^{2+}(1<E<6 \mathrm{keV})$.

Conclusion. - L'émissıon électronique secondaire est un phénomène complexe, en raison de l'influence de nombreux facteurs liés au faisceau ionique et à la cible. Pour un multiplicateur d'électrons donné et dans des conditions expérimentales bien définies, seuls les facteurs inhérents au faisceau ionique sont susceptibles de varier, c'est-à-dire l'énergie, la vitesse, la masse, la nature chimique, la structure moléculaire et la charge de l'ion. Les résultats obtenus dans ces conditions sont résumés ci-après :

- le gain du multiplicateur est une fonction linéaire de l'énergie dans le domaine allant de 1,2 à
$5 \mathrm{keV}$, quelles que soient la nature et la structure de l'ion;

- à énergie égale, le gain varie avec l'inverse de la racine carrée de la masse des isotopes d'un élément :

- à vitesse constante, le gain croît ar sc le numéro atomique $Z$ de la particule incidente'surtout pour $5<Z<10$ ), d'autant plus rapidement que la vitesse est plus élevée ;

- à vitesse égale, les gains mesurés pour un ion polyatomique et un ion monoatomique, ou de structure plus simple, divergent à partir d'un point commun ;

- à vitesse égale, pour un élément $X$ on a toujours $G_{\mathbf{X}^{2+}}<G_{\mathbf{X}^{+}}$; l'écart augmente avec la vitesse.

L'utilisation du multiplicateur d'électrons est maintenant fréquente en spectrométrie de masse, mais la réponse de ce détecteur est sensible à de nombreux facteurs. Il résulte de nos expériences que ces variations sont considérablement diminuées pour des énergies comprises entre 1 et $2 \mathrm{keV}$; le gain du multiplicateur est alors pratiquement constant, quelles que soient les caractéristiques des ions incidents, si toutefois ils ont la même vitesse.

Remerciements. - Les auteurs remercient M. R. Keller qui a assuré le fonctionnement du spectromètre de masse avec compétence et dévouement.

Manuscrit reçu le 24 novembre 1965.

\section{BIBLIOGRAPHIE}

[1] Hagstrum (H. D.), Phys. Rev., 1956, 96, 336.

[2] Parilis (E. S.) et Kishineskir (L. M.), Sos. Phys. Solid State, 1960, 3, 885.

[3] Allen (J. S.), Phys. Res., 1939, 55, 966.

[4] Allen (J. S.), Rev. Sc. Instr., 1947, 18, 739.

[5] Schaetri (N.), Bull. Ass. Suisse Electron., 1954, 23, 989.

[6] Michijima (M.), J. Appl. Phys. Japan, 1962, 1, 110.

[7] Inghram (M. G.), Hayden (R. J.) et Hess (D. C.), Mass Spectrometry in Physics Research, Natl. Bur. Stand., Washington, Circular 522, p. 257.
[8] Higatsberger (M. J.), Demorest (H. L.) et Nier (A. O.), J. Appl. Phys., 1954, 25, 883.

[9] Ploch (W.) et Walcher (W.), Rev. Sc. Instr., 1951, 22, 1028.

[10] Brunnee (C.), Z. Physik, 1957, 147, 161

[11] Sugrura (T.), Bull. Chem. Soc. Japan, 1962, 35, 1257.

[12] Stanton (H. E.), Chupka (W. A.) et Inghram (M. G.), Rev. Sc. Instr., 1956, 27, 109.

[13] Tel'Kovski (V. G.), Izvest. Akad. Nauk (SSSR), Ser. Fiz., 1956, 20, 1179. 